2005-09-01

\title{
Superdirectivity in MIMO systems
}

Michael A. Jensen

jensen@byu.edu

Matthew L. Morris

Jon W. Wallace

wall@ieee.org

Follow this and additional works at: https://scholarsarchive.byu.edu/facpub

Part of the Electrical and Computer Engineering Commons

\section{Original Publication Citation}

Morris, M. L., M. A. Jensen, and J. W. Wallace. "Superdirectivity in MIMO Systems." Antennas and Propagation, IEEE Transactions on 53.9 (25): 285-7

\section{BYU ScholarsArchive Citation}

Jensen, Michael A.; Morris, Matthew L.; and Wallace, Jon W., "Superdirectivity in MIMO systems" (2005). Faculty Publications. 352.

https://scholarsarchive.byu.edu/facpub/352

This Peer-Reviewed Article is brought to you for free and open access by BYU ScholarsArchive. It has been accepted for inclusion in Faculty Publications by an authorized administrator of BYU ScholarsArchive. For more information, please contact ellen_amatangelo@byu.edu. 


\title{
Superdirectivity in MIMO Systems
}

\author{
Matthew L. Morris, Michael A. Jensen, Senior Member, IEEE, and Jon W. Wallace
}

\begin{abstract}
Multiantenna systems such as devices for multiple-input-multiple-output (MIMO) communication can theoretically use array superdirectivity to optimally exploit the propagation channel. In traditional analyses of MIMO systems, such superdirectivity is not observed due to the commonly applied constraint that limits the excitation current magnitudes. However, when an electromagnetically appropriate constraint on the power radiated by the array is applied, the computed capacity can include effects of transmit superdirectivity. A similar result occurs at the receiver for spatially colored noise. This paper formulates the MIMO system capacity under these circumstances and provides a framework for computing this capacity when the level of tolerable superdirectivity (as measured by the superdirectivity $Q$ factor) is constrained. Example computations using the framework illustrate the impact that superdirectivity can have on achievable MIMO system performance.
\end{abstract}

Index Terms-Antenna gain, information theory, multiple-input multiple-output (MIMO) systems.

\section{INTRODUCTION}

$\mathbf{M}$ ULTIPLE-input-multiple-output (MIMO) systems that exploit multiple antennas at both ends of the link have demonstrated clear advantages over single antenna systems when used in multipath propagation environments [1], [2]. Typical analyses of MIMO links use the channel capacity to determine the system upper performance bound for a given propagation channel. Formulations for this capacity specify the properties of the optimal excitation and receive beamformer for the channel under consideration.

When dealing with these optimal excitations or beamforming weights for closely spaced antennas, we must consider the possibility of array superdirectivity behavior [3]-[7] characterized by very high array directivity in preferred directions. While this phenomenon theoretically allows the system to advantageously exploit the propagation channel spatial characteristics, its implementation is typically considered impractical for a variety of reasons [4]. Therefore, analyses of multiantenna systems should include a mechanism for observing the potential impact of superdirectivity and limiting its influence on the system performance.

It appears that superdirectivity in MIMO systems has not yet been considered, likely due to the traditional transmit excitation constraints and assumed receiver noise characteristics. In this paper, we formulate an electromagnetically consistent con-

Manuscript received November 26, 2004; March 5, 2005. This work was supported in part by the National Science Foundation under Information Technology Research Grant CCR-0313056 and in part by the U.S. Army Research Office under Multi-University Research Initiative Grant W911NF-04-1-0224.

The authors are with the Department of Electrical and Computer Engineering, Brigham Young University, Provo, UT 84602 USA (e-mail: jensen@ee.byu.edu).

Digital Object Identifier 10.1109/TAP.2005.854530

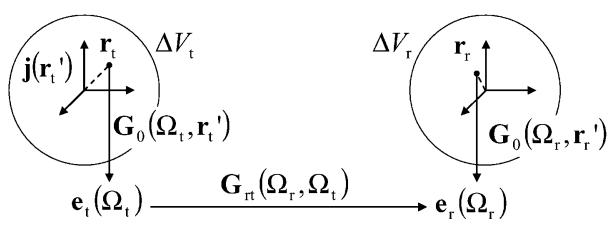

Fig. 1. Basic diagram showing the relevant quantities and coordinates for defining the MIMO channel model.

straint on the system radiated power and a model for noise generated external to the receive array. We demonstrate that these conditions lead to transmit and receive superdirectivity, respectively. We then provide a framework for computing the capacity under these circumstances for cases where the transmitter is aware and unaware of the channel. The formulation includes a mechanism for limiting the superdirectivity effects, as measured by the array $Q$ factor [5]-[7], to within a bound that can be set. The approach is applied to specific examples that highlight the effect of superdirectivity on the capacity bound for multipath channels.

\section{ANALYSIS FRAMEWORK}

Our objective is to illustrate how superdirectivity impacts MIMO system performance and how to assess this performance when the level of tolerable superdirectivity is limited due to practical considerations. We therefore present a communication model wherein the effect of superdirectivity can be observed and formulate the MIMO system capacity when this superdirectivity is constrained. Throughout this discussion, a narrow-band channel is assumed with sinusoidal steady-state variation $(\exp (j \omega t))$. Additionally, boldface uppercase and lowercase letters will describe matrices (matrix $\mathbf{H}$ with $m n$th element $H_{m n}$ ) and column vectors (vector $\mathbf{h}$ with $m$ th element $\left.h_{m}\right)$, respectively.

\section{A. Communication Channel Model}

We will use a generalized communication scenario so that the developed framework can be adapted to specific antenna configurations and propagation environments. Consider an arbitrary propagation channel linked by transmit and receive elements which are confined to the volumes $\Delta V_{\mathrm{t}}$ and $\Delta V_{\mathrm{r}}$, respectively. For simplicity, local coordinate systems are assumed for the transmit space $\left(\mathbf{r}_{\mathrm{t}}\right)$ and receive space $\left(\mathbf{r}_{\mathbf{r}}\right)$. Fig. 1 shows a simple diagram of this scenario.

The $N_{\mathrm{t}}$ transmit antenna elements will be represented by a set of $N_{\mathrm{t}}$ vector basis functions $\tau_{n}$ so that the transmit current distribution is given as

$$
\mathbf{j}\left(\mathbf{r}_{\mathrm{t}}^{\prime}\right)=\sum_{n=1}^{N_{\mathrm{t}}} \boldsymbol{\tau}_{n}\left(\mathbf{r}_{\mathrm{t}}^{\prime}\right) i_{n}
$$


where $i_{n}$ is a complex weight. The vector radiation pattern in the far-field can then be expressed as [8]

$$
\begin{aligned}
\mathbf{e}_{\mathrm{t}}\left(\Omega_{\mathrm{t}}\right) & =\int_{\Delta V_{\mathrm{t}}} d \mathbf{r}_{\mathrm{t}}^{\prime} \mathbf{G}_{0}\left(\Omega_{\mathrm{t}}, \mathbf{r}_{\mathrm{t}}^{\prime}\right) \mathbf{j}_{\mathrm{t}}\left(\mathbf{r}_{\mathrm{t}}^{\prime}\right) \\
& =\sum_{n=1}^{N_{\mathrm{t}}} \underbrace{\left[\int_{\Delta V_{\mathrm{t}}} d \mathbf{r}_{\mathrm{t}}^{\prime} \mathbf{G}_{0}\left(\Omega_{\mathrm{t}}, \mathbf{r}_{\mathrm{t}}^{\prime}\right) \boldsymbol{\tau}_{n}\left(\mathbf{r}_{\mathrm{t}}^{\prime}\right)\right]}_{\mathbf{e}_{\mathrm{t} n}\left(\Omega_{\mathrm{t}}\right)} i_{n} \\
& =\mathbf{E}_{\mathrm{t}}\left(\Omega_{\mathrm{t}}\right) \mathbf{i}
\end{aligned}
$$

where $\Omega_{\mathrm{t}}$ represents a direction in solid angle with elevation and azimuth angles $\theta_{\mathrm{t}}$ and $\phi_{\mathrm{t}}$, respectively. The function $\mathbf{e}_{\mathrm{t} n}\left(\Omega_{\mathrm{t}}\right)$, which is the $n$th column of $\mathbf{E}_{\mathrm{t}}\left(\Omega_{\mathrm{t}}\right)$, represents the radiation pattern of the $n$th basis function for unit driving current $\left(i_{n}=1\right)$ with all other basis functions open-circuited $\left(i_{q}=0\right.$ for $\left.q \neq n\right)$. The dyadic Green's function $\mathbf{G}_{0}$ relates the current distribution to the radiated fields in the far-zone and is normalized by the spherical wave factor $e^{-j k_{0} r_{\mathrm{t}}} / r_{\mathrm{t}}$, with $k_{0}$ the free-space wavenumber, so that the transmitted field pattern depends only on the observation angle.

After propagation through the channel, the field is received by $N_{\mathrm{r}}$ antennas represented by the $N_{\mathrm{r}}$ vector basis functions $\rho_{m}$ each with far-field radiation pattern

$$
\mathbf{e}_{\mathrm{r} m}\left(\Omega_{\mathrm{r}}\right)=\int_{\Delta V_{\mathrm{r}}} d \mathbf{r}_{\mathrm{r}}^{\prime} \mathbf{G}_{0}\left(\Omega_{\mathrm{r}}, \mathbf{r}_{\mathrm{r}}^{\prime}\right) \boldsymbol{\rho}_{m}\left(\mathbf{r}_{\mathrm{r}}^{\prime}\right)
$$

If $\mathbf{G}_{\mathrm{rt}}\left(\Omega_{\mathrm{r}}, \Omega_{\mathrm{t}}\right)$ represents the dyadic gain function relating the field radiated at angle $\Omega_{\mathrm{t}}$ and received at angle $\Omega_{\mathrm{r}}$, the voltage received by the $m$ th sensor function is

$$
v_{m}=\oint_{\Omega_{\mathrm{r}}} d \Omega_{\mathrm{r}} \oint_{\Omega_{\mathrm{t}}} d \Omega_{\mathrm{t}} \mathbf{e}_{\mathrm{r} m}^{T}\left(\Omega_{\mathrm{r}}\right) \mathbf{G}_{\mathrm{rt}}\left(\Omega_{\mathrm{r}}, \Omega_{\mathrm{t}}\right) \mathbf{e}_{\mathrm{t}}\left(\Omega_{\mathrm{t}}\right)+\eta_{m}
$$

where the integrations are over spheres surrounding the transmit and receive spaces, $\{\cdot\}^{T}$ is a transpose, and $\eta_{m}$ is noise. Substitution of (3) into (5) yields the linear system

$$
\mathbf{v}=\mathbf{H i}+\boldsymbol{\eta}
$$

where the discrete transfer matrix elements are given as

$$
H_{m n}=\oint_{\Omega_{\mathrm{r}}} d \Omega_{\mathrm{r}} \oint_{\Omega_{\mathrm{t}}} d \Omega_{\mathrm{t}} \mathbf{e}_{\mathrm{r} m}^{T}\left(\Omega_{\mathrm{r}}\right) \mathbf{G}_{\mathrm{rt}}\left(\Omega_{\mathrm{r}}, \Omega_{\mathrm{t}}\right) \mathbf{e}_{\mathrm{t} n}\left(\Omega_{\mathrm{t}}\right) .
$$

\section{B. Superdirectivity}

With the communication model of (6), we are prepared to discuss the superdirectivity characteristics of the transmit and receive arrays. There are a variety of practical problems associated with superdirectivity excitations, including high antenna currents (which lead to high ohmic loss), extreme sensitivity to the excitation weights, and narrow operating bandwidth [4]. While metrics exist for quantifying the level of superdirectivity associated with an array excitation in terms of these practical impacts, we seek a metric that is closely tied to the multiantenna capacity formulation. We will see that the superdirectivity geometric $Q$ factor [5]-[7], which quantifies the usable bandwidth of the array, is a particularly convenient metric for this analysis. To compute this metric for the transmit array, we construct the Hermitian matrix

$$
\mathbf{B}_{\mathrm{t}}=\oint_{\Omega_{\mathrm{t}}} d \Omega_{\mathrm{t}} \mathbf{E}_{\mathrm{t}}^{\dagger}\left(\Omega_{\mathrm{t}}\right) \mathbf{E}_{\mathrm{t}}\left(\Omega_{\mathrm{t}}\right)
$$

where $\{\cdot\}^{\dagger}$ is the matrix conjugate transpose. Assuming that all basis functions $\tau_{n}$ are identical other than their positions in space, we can normalize this matrix to have unit entries along the diagonal as

$$
\mathbf{A}_{\mathrm{t}}=\mathbf{B}_{\mathrm{t}} / B_{\mathrm{t}, 11} .
$$

The superdirectivity $Q$ factor for this array for a vector of transmit currents $\mathbf{i}$ is then given as

$$
Q_{\mathrm{t}}=\frac{\mathbf{i}^{\dagger} \mathbf{i}}{\mathbf{i}^{\dagger} \mathbf{A}_{\mathrm{t}} \mathbf{i}}
$$

The product of $Q_{\mathrm{t}}$ and the quality factor $Q_{e}$ of the individual array elements represents the quality factor of the antenna array for the excitation vector i [3]. Therefore, a high $Q$ factor corresponds to a small usable bandwidth. For example, suppose we use an element that has $Q_{e}=10$ when operating in isolation. This corresponds to a $10 \%$ frequency bandwidth, something easily obtainable by practical elements such as a half-wave dipole. If the array configuration leads to a modest $Q$ factor of ten, the overall array quality factor will be 100 , leading to a frequency bandwidth of only $1 \%$. Therefore, the attempt to use superdirectivity to enhance system performance will in most cases fail due to this bandwidth reduction (in addition to the other practical difficulties outlined previously). Since the goal of using MIMO technology is to obtain high spectral efficiency, this severe bandwidth reduction can be considered counterproductive to this fundamental goal.

When the array is used for information communication, the excitation vector $\mathbf{i}$ and therefore $Q_{\mathrm{t}}$ will be time-variant. We can, however, gain insight into the value $Q_{\mathrm{t}}$ from $\mathbf{A}_{\mathrm{t}}$ (which depends only on the array properties). Let the eigenvalue decomposition (EVD) of $\mathbf{A}_{\mathrm{t}}$ be represented by $\mathbf{A}_{\mathrm{t}}=\boldsymbol{\xi}_{\mathrm{t}} \boldsymbol{\Lambda}_{\mathrm{t}} \boldsymbol{\xi}_{\mathrm{t}}^{\dagger}$ [9], where $\xi_{\mathrm{t}}$ is a unitary matrix of eigenvectors and $\boldsymbol{\Lambda}_{\mathrm{t}}$ is a diagonal matrix of real eigenvalues (since $\mathbf{A}_{t}$ is Hermitian). If we expand the current at time $t$ using the eigenvectors as $\mathbf{i}(t)=\boldsymbol{\xi}_{\mathrm{tn}} \boldsymbol{\alpha}(t)$, where $\boldsymbol{\alpha}(t)$ is a vector of weights, then $Q_{\mathrm{t}}(t)=\boldsymbol{\alpha}^{\dagger}(t) \boldsymbol{\alpha}(t) / \boldsymbol{\alpha}^{\dagger}(t) \boldsymbol{\Lambda}_{\mathrm{t}} \boldsymbol{\alpha}(t)$. $Q_{\mathrm{t}}$ will therefore be large (indicative of superdirectivity) when the current is aligned with eigenvectors associated with small eigenvalues, and reaches a maximum value of $Q_{\mathrm{t}, \max }=1 / \Lambda_{\mathrm{t}, \min }$, where $\Lambda_{\mathrm{t}, \min }$ represents the smallest eigenvalue.

For the following, we will define $Q_{\mathrm{to}}$ as the highest $Q$ factor that we will tolerate for the transmit array. The matrix $\hat{\xi}_{\mathrm{t}}$ represents the eigenvectors in $\xi_{\mathrm{t}}$ associated with eigenvalues in $\boldsymbol{\Lambda}_{\mathrm{t}}$ that are greater than $1 / Q_{\mathrm{t} 0}$. Similarly, we can construct the matrix $\mathbf{A}_{\mathrm{r}}$ using (8) and (9) with the replacements $\mathbf{e}_{\mathrm{t} n} \leftarrow \mathbf{e}_{\mathrm{r} m}$ and $\Omega_{\mathrm{t}} \leftarrow \Omega_{\mathrm{r}}$. Using the EVD $\mathbf{A}_{\mathrm{r}}=\xi_{\mathrm{r}} \boldsymbol{\Lambda}_{\mathrm{r}} \xi_{\mathrm{r}}^{\dagger}$ and defining the threshold $Q_{\mathrm{r} 0}, \hat{\boldsymbol{\xi}}_{\mathrm{r}}$ represents the eigenvectors in $\xi_{\mathrm{r}}$ associated with eigenvalues in $\boldsymbol{\Lambda}_{\mathrm{r}}$ that are greater than $1 / Q_{\mathrm{r} 0}$. 


\section{Noise and Receive Superdirectivity}

Noise can be introduced by sources external to the receiver (interference) and by components within the receiver (thermal noise). We will first consider the case of external interference, where a noise field $\mathbf{n}\left(\Omega_{\mathrm{r}}\right)$ impinges on the receiver. The noise vector contains zero-mean complex Gaussian random variables where the contributions from different directions are uncorrelated, leading to the expression

$$
\mathrm{E}\left\{\mathbf{n}\left(\Omega_{\mathrm{r}}\right) \mathbf{n}^{\dagger}\left(\Omega_{\mathrm{r}}^{\prime}\right)\right\}=\sigma_{\eta}^{2} p_{\eta}\left(\Omega_{\mathrm{r}}\right) \delta\left(\Omega_{\mathrm{r}}-\Omega_{\mathrm{r}}^{\prime}\right) \mathbf{I}
$$

where $\mathrm{E}\{\cdot\}$ is an expectation, $\mathbf{I}$ is the identity matrix, $\sigma_{\eta}^{2} p_{\eta}\left(\Omega_{r}\right)$ represents the noise angular power spectrum, and $\delta(\cdot)$ is the Dirac delta function. According to the convention presented in (5), the vector of received noise voltages is

$$
\boldsymbol{\eta}=\oint_{\Omega_{\mathrm{r}}} d \Omega_{\mathrm{r}} \mathbf{E}_{\mathrm{r}}^{T}\left(\Omega_{\mathrm{r}}\right) \mathbf{n}\left(\Omega_{\mathrm{r}}\right)
$$

where $\mathbf{e}_{\mathrm{r} m}\left(\Omega_{\mathrm{r}}\right)$ forms the $m$ th column of the matrix $\mathbf{E}_{\mathrm{r}}\left(\Omega_{\mathrm{r}}\right)$. Using this result with (11), the noise covariance $\mathbf{R}_{\eta}=\mathrm{E}\left\{\boldsymbol{\eta} \boldsymbol{\eta}^{\dagger}\right\}$ is given as

$$
\mathbf{R}_{\eta}=\sigma_{\eta}^{2} \oint_{\Omega_{\mathrm{r}}} d \Omega_{\mathrm{r}} \mathbf{E}_{\mathrm{r}}^{T}\left(\Omega_{\mathrm{r}}\right) \mathbf{E}_{\mathrm{r}}^{*}\left(\Omega_{\mathrm{r}}\right) p_{\eta}\left(\Omega_{\mathrm{r}}\right) .
$$

To accommodate this nondiagonal (spatially colored) noise covariance matrix, it is customary to prewhiten the signal using the beamformer $\mathbf{R}_{\eta}^{-1 / 2} \mathbf{v}$ [10]. If, at a given time instant, the vector $\mathbf{v}$ is aligned with an eigenvector of $\mathbf{R}_{\eta}$ corresponding to a small eigenvalue, then the beamformer will create a large signal gain. However, we recognize that $\mathbf{R}_{\eta}$ in (13) is very similar to $\mathbf{B}_{\mathbf{r}}$ computed using appropriate substitutions in (8). In fact, if $p_{\eta}\left(\Omega_{\mathrm{r}}\right)=1$, then $\mathbf{R}_{\eta}=\sigma_{\eta}^{2} \mathbf{B}_{\mathrm{r}}^{*}$, indicating that this gain represents superdirectivity. Physically, the array is using superdirectivity to attenuate the external noise power while maintaining high gain for the desired signal. This will only occur when the noise and signal arrivals are characterized by different angular power distributions.

Our goal is to ensure that the system does not try to exploit superdirectivity beyond a predetermined level. Using our definitions in Section II-B, we therefore first apply the beamformer represented by $\hat{\xi}_{\mathrm{r}}^{\dagger}$ to create the signal

$$
\mathbf{v}_{0}=\hat{\xi}_{\mathrm{r}}^{\dagger} \mathbf{v}=\hat{\xi}_{\mathrm{r}}^{\dagger} \mathbf{H i}+\hat{\xi}_{\mathrm{r}}^{\dagger} \boldsymbol{\eta}
$$

where the noise $\hat{\boldsymbol{\eta}}=\hat{\boldsymbol{\xi}}_{\mathrm{r}}^{\dagger} \boldsymbol{\eta}$ has covariance $\hat{\mathbf{R}}_{\eta}=\hat{\boldsymbol{\xi}}_{\mathrm{r}}^{\dagger} \mathbf{R}_{\eta} \hat{\boldsymbol{\xi}}_{\mathrm{r}}$. With this projection, any subsequently applied receive beamforming weights characterized by a $Q$ factor above $Q_{\mathrm{r} 0}$ will lie in the null-space of $\mathbf{v}_{0}$ and therefore will not contribute to the capacity. Application of the prewhitening filter gives

$$
\hat{\mathbf{v}}=\hat{\mathbf{R}}_{\eta}^{-1 / 2} \mathbf{v}_{0}=\underbrace{\hat{\mathbf{R}}_{\eta}^{-1 / 2} \hat{\boldsymbol{\xi}}_{\mathrm{r}}^{\dagger} \mathbf{H}}_{\mathbf{H}_{0}} \mathbf{i}+\hat{\mathbf{R}}_{\eta}^{-1 / 2} \hat{\boldsymbol{\eta}}
$$

where the noise $\hat{\mathbf{R}}_{\eta}^{-1 / 2} \hat{\boldsymbol{\eta}}$ has covariance $\mathbf{I}$.

When the dominant noise source is thermal noise generated in the receiver front-end, we typically assume that the vector $\boldsymbol{\eta}$ consists of zero-mean complex Gaussian elements with covariance
$\mathbf{R}_{\eta}=\sigma_{\eta}^{2} \mathbf{I}$. A beamformer applied to the received signal plus noise can no longer suppress this spatially white noise through selective spatial filtering. Mathematically, we observe that the prewhitening beamformer is simply a scaled identity matrix and therefore will not introduce small eigenvalues (and therefore superdirectivity).

\section{Radiated Power Constraint and Transmit Superdirectivity}

A traditional transmit power constraint for the system represented in (6) would be [11]

$$
\mathrm{E}\left\{\mathbf{i}^{\dagger} \mathbf{i}\right\}=\operatorname{Tr}\left(\mathbf{R}_{\mathrm{t}}\right) \leq P_{T}
$$

where $\mathbf{R}_{\mathrm{t}}=\mathrm{E}\left\{\mathbf{i i}^{\dagger}\right\}$ and $\operatorname{Tr}(\cdot)$ represents a trace. However, (16) does not represent the actual power radiated by the currents, which is typically the quantity constrained by regulating agencies. To properly formulate this radiated power, we use (3) to compute the radiated power averaged over one sinusoidal cycle (assuming the complex envelope of the current remains constant over this interval) as

$$
\begin{aligned}
p_{\text {rad }} & =\frac{1}{2 Z_{0}} \oint_{\Omega_{\mathrm{t}}} d \Omega_{\mathrm{t}} \mathbf{e}_{\mathrm{t}}^{\dagger}\left(\Omega_{\mathrm{t}}\right) \mathbf{e}_{\mathrm{t}}\left(\Omega_{\mathrm{t}}\right) \\
& =\mathbf{i}^{\dagger} \underbrace{\left[\frac{1}{2 Z_{0}} \oint_{\Omega_{\mathrm{t}}} d \Omega_{\mathrm{t}} \mathbf{E}_{\mathrm{t}}^{\dagger}\left(\Omega_{\mathrm{t}}\right) \mathbf{E}_{\mathrm{t}}\left(\Omega_{\mathrm{t}}\right)\right]}_{\mathbf{z}_{\mathrm{t}}=\mathbf{B}_{\mathrm{t}} / 2 Z_{0}} \mathbf{i}
\end{aligned}
$$

where $Z_{0}$ is the intrinsic impedance of free space. The average radiated power obtained by taking the expectation of (17) is

$$
P_{\text {rad }}=\operatorname{Tr}\left(\mathbf{R}_{\mathrm{t}} \mathbf{Z}_{\mathrm{t}}\right)=\operatorname{Tr} \underbrace{\left(\mathbf{Z}_{\mathrm{t}}^{1 / 2} \mathbf{R}_{\mathrm{t}} \mathbf{Z}_{\mathrm{t}}^{1 / 2}\right)}_{\hat{\mathbf{R}}_{\mathrm{t}}} \leq P_{T}
$$

where the last inequality represents the constraint placed on the radiated power.

The effect of the radiated power constraint of (18) can be readily observed when it is applied to determine the system capacity. For the communications model in (15), the mutual information between $\hat{\mathbf{v}}$ and $\mathbf{i}$ satisfies [12]

$$
I(\hat{\mathbf{v}}, \mathbf{i}) \leq \log _{2} \operatorname{det}\left(\mathbf{H}_{0} \mathbf{R}_{\mathrm{t}} \mathbf{H}_{0}^{\dagger}+\mathbf{I}\right)
$$

where $\operatorname{det}(\cdot)$ is a determinant and equality occurs when $\mathbf{i}$ is drawn from a complex Gaussian constellation. Observing from (18) that $\mathbf{R}_{\mathrm{t}}=\mathbf{Z}_{\mathrm{t}}^{-1 / 2} \hat{\mathbf{R}}_{\mathrm{t}} \mathbf{Z}_{\mathrm{t}}^{-1 / 2}$ and substituting this result into (19) shows how the small eigenvalues in $\mathbf{Z}_{\mathrm{t}}$ can lead to spatial channels with high gain. Since $\mathbf{Z}_{\mathrm{t}}=\mathbf{B}_{\mathrm{t}} / 2 Z_{0}$, it is evident that this condition corresponds to transmit superdirectivity. Physically, the multiplication by $\mathbf{Z}_{\mathrm{t}}^{-1 / 2}$ operates as a transmit beamformer that directs power into angular directions with high channel gain.

It is important to emphasize that since the traditional power constraint does not limit the radiated power, it does not penalize a solution that puts power into the directions that do not strongly couple to the receiver. For this reason, its use does not typically lead to the transmit superdirectivity introduced as a result of the radiated power constraint of (18). 
We can limit the transmit superdirectivity to have a $Q$ factor below $Q_{\mathrm{t} 0}$ by requiring the excitation $\mathrm{i}$ to remain within the subspace spanned by $\hat{\xi}_{\mathrm{t}}$. To enforce this constraint, we parameterize the covariance $\mathbf{R}_{\mathrm{t}}$ using the basis $\hat{\xi}_{\mathrm{t}}$ as

$$
\mathbf{R}_{\mathrm{t}}=\hat{\xi}_{\mathrm{t}} \mathbf{R}_{0} \hat{\xi}_{\mathrm{t}}^{\dagger}
$$

where $\mathbf{R}_{0}$ is a positive semidefinite matrix. From (18)

$$
\operatorname{Tr}\left(\hat{\mathbf{R}}_{\mathrm{t}}\right)=\operatorname{Tr}\left(\hat{\boldsymbol{\xi}}_{\mathrm{t}} \mathbf{R}_{0} \hat{\boldsymbol{\xi}}_{\mathrm{t}}^{\dagger} \mathbf{Z}_{\mathrm{t}}\right)=\operatorname{Tr}\left(\hat{\mathbf{Z}}_{\mathrm{t}}^{1 / 2} \mathbf{R}_{0} \hat{\mathbf{Z}}_{\mathrm{t}}^{1 / 2}\right)
$$

where $\hat{\mathbf{Z}}_{\mathrm{t}}=\hat{\boldsymbol{\xi}}_{\mathrm{t}}^{\dagger} \mathbf{Z}_{\mathrm{t}} \hat{\boldsymbol{\xi}}_{\mathrm{t}}$, which leads to $\mathbf{R}_{0}=\hat{\mathbf{Z}}_{\mathrm{t}}^{-1 / 2} \hat{\mathbf{R}}_{\mathrm{t}} \hat{\mathbf{Z}}_{\mathrm{t}}^{-1 / 2}$. Using this result and (20) in (19) leads to

$$
I(\hat{\mathbf{v}}, \mathbf{i}) \leq \log _{2} \operatorname{det}\left(\hat{\mathbf{H}} \hat{\mathbf{R}}_{\mathrm{t}} \hat{\mathbf{H}}^{\dagger}+\mathbf{I}\right)
$$

where

$$
\hat{\mathbf{H}}=\hat{\mathbf{R}}_{\eta}^{-1 / 2} \hat{\boldsymbol{\xi}}_{\mathrm{r}}^{\dagger} \mathbf{H} \hat{\boldsymbol{\xi}}_{\mathrm{t}} \hat{\mathbf{Z}}_{\mathrm{t}}^{-1 / 2} .
$$

We point out that since $\mathbf{Z}_{\mathrm{t}}$ and $\mathbf{A}_{\mathrm{t}}$ share the same eigenvectors, $\hat{\mathbf{Z}}_{\mathrm{t}}=\hat{\mathbf{\Lambda}}_{Z}$, which represents the diagonal matrix of eigenvalues of $\mathbf{Z}_{\mathrm{t}}$ corresponding to the eigenvectors in $\hat{\xi}_{\mathrm{t}}$.

\section{E. Capacity}

Equation (22) provides a concise mutual information expression that can be maximized to determine the system capacity under the applied constraints. Under the assumption that the transmitter is aware of the channel transfer matrix $\mathbf{H}$, the covariance $\hat{\mathbf{R}}_{\mathrm{t}}$ that maximizes capacity can be constructed from the water-filling solution [2], [11] using the power constraint $\operatorname{Tr}\left(\hat{\mathbf{R}}_{\mathrm{t}}\right) \leq P_{T}$ from (18).

For scenarios where the transmitter is unaware of the channel matrix, we use $\hat{\mathbf{R}}_{\mathrm{t}}=\left(P_{T} / \hat{N}_{\mathrm{t}}\right) \mathbf{I}$, where $\hat{N}_{\mathrm{t}}$ is the dimensionality of $\hat{\mathbf{R}}_{\mathrm{t}}$ [1], [11]. Placing this covariance in (22) leads to the uninformed transmitter capacity

$$
C_{\mathrm{ut}}=\log _{2} \operatorname{det}\left(\frac{P_{T}}{\hat{N}_{\mathrm{t}}} \hat{\mathbf{H}} \hat{\mathbf{H}}^{\dagger}+\mathbf{I}\right) .
$$

We note that the approach used in this paper is overly restrictive since there may exist excitations or beamforming weights not confined to the subspaces spanned by $\hat{\xi}_{\mathrm{t}}$ and $\hat{\xi}_{\mathrm{r}}$ that lead to $Q$ factors below the specified thresholds. Unfortunately, it is not clear how to formulate linear constraints on the transmit and receive covariances that allow these excitations, and we will therefore proceed with the analysis as formulated.

\section{F. Signal Correlation}

It is noteworthy that the effective transmit and receive beamformers observed in (23) can impact the correlation between the channel matrix elements. For example, the elements of $\mathbf{H}$ corresponding to closely spaced antenna elements will generally be highly correlated. However, because superdirectivity can create effective beams that are nearly orthogonal, the elements of $\hat{\mathbf{H}}$ may have reduced correlation.

We will assess this impact for uniform linear arrays by assuming that the channel matrix elements are zero mean and that $H_{m n}$ indicates the transfer function between elements at $\mathbf{r}_{\mathrm{t} n}=(n d, 0,0)$ and $\mathbf{r}_{\mathrm{r} m}=(m d, 0,0)$, where $d$ is the element spacing. The transmit and receive correlations taken at the antenna terminals are

$$
\begin{aligned}
& X_{\mathrm{t}, q}=\frac{1}{N_{\mathrm{r}} N_{q}} \sum_{n=1}^{N_{q}} \sum_{m=1}^{N_{\mathrm{r}}} \mathrm{E}\left\{H_{m n} H_{m, n+q}^{*}\right\} \\
& X_{\mathrm{r}, p}=\frac{1}{N_{\mathrm{t}} N_{p}} \sum_{n=1}^{N_{\mathrm{t}}} \sum_{m=1}^{N_{p}} \mathrm{E}\left\{H_{m n} H_{m+p, n}^{*}\right\}
\end{aligned}
$$

where $N_{q}\left(N_{p}\right)$ represents the number of unique pairs of transmit (receive) antennas separated by a distance $q d(p d)$. The expectation can be approximated by an average taken over an ensemble of channel matrix realizations. The transmit and receive correlation coefficients are then constructed using $\rho_{\mathrm{t}, q}=X_{\mathrm{t}, q} / X_{\mathrm{t}, 0}$ and $\rho_{\mathrm{r}, p}=X_{\mathrm{r}, p} / X_{\mathrm{r}, 0}$, respectively. The correlation coefficients at the input of the transmit beamformer and output of the receive beamformer can be computed by the same procedure with $\hat{\mathbf{H}}$ used in place of $\mathbf{H}$.

\section{REPRESENTATIVE APPLICATION}

The derivations in Section II provide a general framework for determining the capacity of an MIMO system under the constraint that superdirectivity is limited. It is instructive to now apply the technique to a specific set of transmit and receive basis functions. For simplicity, we will consider two-dimensional arrays of Hertzian dipoles at the transmit and receive coupled with a two-dimensional, single-polarization description of the propagation channel. The arrays at transmit and receive will be identical.

The use of Hertzian dipoles has some consequences that must be understood. First, such elements themselves have infinite quality factor, which means they cannot be used over any bandwidth. However, since the superdirectivity $Q$ factor represents the quality factor created by the array geometry and does not account for the element quality factor, these elements will allow us to observe the superdirectivity behavior. Because half-wave dipoles have radiation patterns similar to those of the Hertzian dipole, the results obtained here will be representative of what would be observed for the more practical longer dipole antenna. Second, because the Hertzian dipole does not suffer current deformations due to mutual coupling and since the formulation here uses open-circuit voltages to represent the received signal (so that mutual impedances do not enter into the formulation), we can assess the impact of superdirectivity without the added complication of the effect of electromagnetic coupling. Such coupling effects can be included if desired using the alternate formulation in Section IV. This model problem therefore provides computational simplicity while demonstrating the key features of MIMO systems impacted by superdirectivity effects.

\section{A. Basis Functions and Channel Description}

Because we are operating in a single polarization environment, the basis functions can be expressed as scalars. The Hertzian dipoles are represented as $\tau_{n}\left(\mathbf{r}_{\mathrm{t}}\right)=\delta\left(\mathbf{r}_{\mathrm{t}}-\mathbf{r}_{\mathrm{t} n}\right)$ and $\rho_{m}\left(\mathbf{r}_{\mathrm{r}}\right)=\delta\left(\mathbf{r}_{\mathrm{r}}-\mathbf{r}_{\mathrm{r} m}\right)$, where $\mathbf{r}_{\mathrm{t} n}=\left(x_{\mathrm{t} n}, y_{\mathrm{t} n}, 0\right)$ and $\mathbf{r}_{\mathrm{r} m}=\left(x_{\mathrm{r} m}, y_{\mathrm{r} m}, 0\right)$ indicate the locations of the $n$th transmit 
and $m$ th receive dipole, respectively. With this basis, we can use the (normalized) free-space scalar Green's function [8]

$$
\begin{aligned}
G_{0}(\Omega, \mathbf{r}) & =\sin \theta \psi(\mathbf{r}, \Omega) \\
\psi(\mathbf{r}, \Omega) & =e^{j k_{0}(x \sin \theta \cos \phi+y \sin \theta \sin \phi+z \cos \theta)} .
\end{aligned}
$$

The radiation patterns at transmit and receive become

$$
\begin{aligned}
e_{\mathrm{t} n}\left(\Omega_{\mathrm{t}}\right) & =\sin \theta_{\mathrm{t}} \psi\left(\mathbf{r}_{\mathrm{t} n}, \Omega_{\mathrm{t}}\right) \\
e_{\mathrm{r} m}\left(\Omega_{\mathrm{r}}\right) & =\sin \theta_{\mathrm{r}} \psi\left(\mathbf{r}_{\mathrm{r} m}, \Omega_{\mathrm{r}}\right) .
\end{aligned}
$$

We next assume a directional channel model consisting of $L$ plane waves confined to the horizontal plane with the $\ell$ th plane wave characterized by departure direction $\Omega_{\mathrm{t} \ell}=\left(\theta_{\mathrm{t} \ell}=\right.$ $\left.\pi / 2, \phi_{\mathrm{t} \ell}\right)$, arrival direction $\Omega_{\mathrm{r} \ell}=\left(\theta_{\mathrm{r} \ell}=\pi / 2, \phi_{\mathrm{r} \ell}\right)$, and complex gain $\beta_{\ell}$. In this analysis, the plane waves are grouped into angular clusters at transmit and receive, with the angles of the clusters distributed uniformly in the horizontal plane and the angles of the arrivals within a cluster satisfying a Laplacian distribution. The complex gains $\beta_{\ell}$ are drawn from a complex Gaussian distribution. The details of these distributions as well as a detailed demonstration of the model accuracy are provided in [13]. For our single-polarization environment, the path-based channel can be expressed as

$$
G_{\mathrm{rt}}\left(\Omega_{\mathrm{r}}, \Omega_{\mathrm{t}}\right)=\sum_{\ell=1}^{L} \beta_{\ell} \delta\left(\Omega_{\mathrm{r}}-\Omega_{\mathrm{r} \ell}\right) \delta\left(\Omega_{\mathrm{t}}-\Omega_{\mathrm{t} \ell}\right)
$$

which when used in (7) leads to

$$
H_{m n}=\sum_{\ell=1}^{L} \beta_{\ell} \psi\left(\mathbf{r}_{\mathrm{r} m}, \Omega_{\mathrm{r} \ell}\right) \psi\left(\mathbf{r}_{\mathrm{t} n}, \Omega_{\mathrm{t} \ell}\right) .
$$

To construct $\mathbf{B}_{\mathrm{t}}$, we use (8) with our basis to obtain

$$
B_{\mathrm{t}, n q}=2 \pi \int_{0}^{\pi} d \theta_{\mathrm{t}} J_{0}\left(k_{0} \sin \theta_{\mathrm{t}}\left|\mathbf{r}_{\mathrm{t} n}-\mathbf{r}_{\mathrm{t} q}\right|\right) \sin ^{3} \theta_{\mathrm{t}}
$$

where $|\cdot|$ represents the magnitude and $J_{0}(\cdot)$ is the zerothorder Bessel function. For identical arrays as used here, $\mathbf{B}_{\mathrm{r}}=$ $\mathbf{B}_{\mathrm{t}}$. When considering external noise, we will assume it arrives isotropically so that $p_{\eta}\left(\Omega_{\mathrm{r}}\right)=1$ leading to $\mathbf{R}_{\eta}=\sigma_{\eta}^{2} \mathbf{B}_{\mathrm{r}}^{*}$. When thermal noise is assumed, $\mathbf{R}_{\eta}=\sigma_{\eta}^{2} \mathbf{I}$.

We emphasize that the general conclusions we will draw are not strongly tied to our choices for channel and noise models. As long as these models 1) lead to variation in the power transferred to the receiver as a function of the transmit (departure) angle and 2) create different angular distributions for the received signal and noise, the effects of superdirectivity will be observed. Therefore, the focus in the following is more on the observed trends than the absolute capacity numbers, which are strongly dependent on the models used.

\section{B. Signal-to-Noise Ratio}

To compute the MIMO system capacity, we must specify the channel average single-input single-output (SISO) signal-tonoise ratio (SNR). To accomplish this, we first construct the scalar $B_{\mathrm{t}}$ for a single dipole using (33) and set $Z_{\mathrm{t}}=B_{\mathrm{t}} / 2 Z_{0}$ so that, for a single transmit antenna, the average radiated power

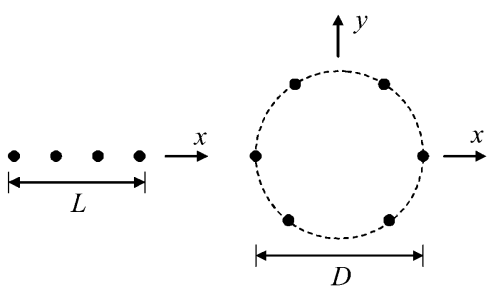

Fig. 2. Uniform linear and circular arrays of $z$-oriented Hertzian dipoles used in the computations.

will be $P_{T}=|i|^{2} Z_{\mathrm{t}}$. For thermal noise where the single-receiver noise power is $\sigma_{\eta}^{2}$, the SISO SNR averaged over all pairs of transmit and receive antennas is [2], [14]

$$
\mathrm{SNR}_{\text {thermal }}=\frac{P_{T}}{Z_{\mathrm{t}} \sigma_{\eta}^{2}} \frac{1}{N_{\mathrm{r}} N_{\mathrm{t}}}\|\mathbf{H}\|_{F}^{2}
$$

where $\|\cdot\|_{F}$ is the matrix Frobenius norm. For the isotropic noise field, we use $R_{\eta}=\sigma_{\eta}^{2} B_{\mathrm{t}}$ to obtain the SISO SNR

$$
\mathrm{SNR}_{\text {isotropic }}=\frac{P_{T}}{Z_{\mathrm{t}} B_{\mathrm{t}} \sigma_{\eta}^{2}} \frac{1}{N_{\mathrm{r}} N_{\mathrm{t}}}\|\mathbf{H}\|_{F}^{2} .
$$

In either case, we set $\sigma_{\eta}^{2}$ to produce the desired SISO SNR for each channel realization. This value is then used to construct the noise covariance as outlined in Section II-C.

\section{Example Computations}

In the following computations, we consider uniform circular arrays with circle diameter $D$ and uniform linear arrays with length $L$, as illustrated in Fig. 2. The array spacing for all arrays is smaller than what might be encountered in typical applications in order to emphasize the superdirectivity effects. The SISO SNR is set to $20 \mathrm{~dB}$. When Monte Carlo simulations are used, 500 realizations of the stochastic channel model are generated, and the results displayed represent the average over the realizations.

We first consider a 16-element uniform circular transmit array with $D=\lambda / 2$, where $\lambda$ is the free-space wavelength. As discussed in Section II-B, the inverse of each eigenvalue of $\mathbf{A}_{\mathrm{t}}$ represents the $Q$ factor associated with an excitation that equals the corresponding eigenvector. Fig. 3, which plots these values, demonstrates the large range of $Q$ factors possible for this array. Certainly, the larger values observed in this plot will not permit transmission of waveforms with even a modest bandwidth. This fact, coupled with the other problems associated with superdirectivity, motivates the need to assess the performance of multiantenna systems when the effect of superdirectivity is constrained.

It is next interesting to explore the impact of the radiated power constraint on the capacity. Using eight-element uniform circular transmit and receive arrays, we compute the capacity and the optimal transmit covariance $\mathbf{R}_{\mathrm{t}}$ for each of the 500 channel realizations using the water-filling solution with the traditional $\left(\operatorname{Tr}\left(\mathbf{R}_{\mathrm{t}}\right) \leq P_{T}\right)$ and modified $\left(\operatorname{Tr}\left(\mathbf{R}_{\mathrm{t}} \mathbf{Z}_{\mathrm{t}}\right) \leq P_{T}\right)$ power constraints. We also compute the radiated power $\operatorname{Tr}\left(\mathbf{R}_{\mathrm{t}} \mathbf{Z}_{\mathrm{t}}\right)$ for each solution. The average capacity and radiated power for thermal noise and $Q_{\mathrm{t} 0}=Q_{\mathrm{r} 0}=10^{100}$ are shown 


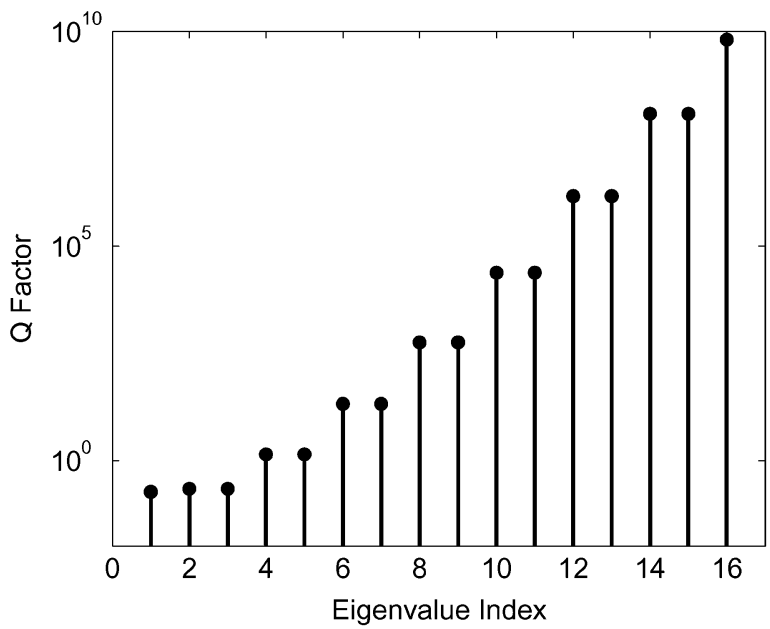

Fig. 3. $Q$ factors associated with each of the eigenvectors of $\mathbf{A}_{\mathbf{t}}$ for a 16-element uniform circular array with diameter $D=\lambda / 2$.
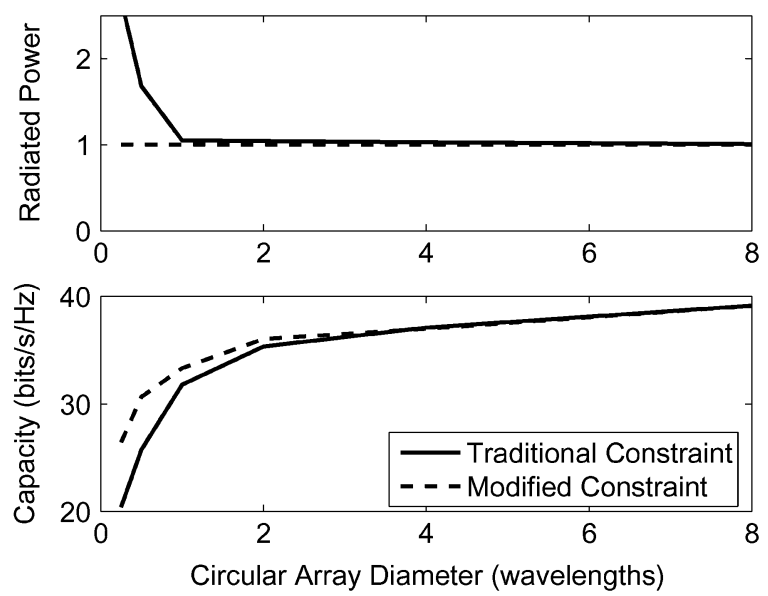

Fig. 4. Radiated power and capacity versus array diameter for the eight-element uniform circular array computed using the water-filling solution with traditional and modified power constraints. The results represent averages over 500 channel realizations.

in Fig. 4 for $P_{T}=1$. For compact arrays, the traditional constraint leads to much higher radiated power than specified while the modified constraint produces the proper power. However, because the modified constraint enables superdirectivity, the capacity for this case exceeds that achieved by the traditional constraint. As the array element spacing increases, $\mathbf{Z}_{\mathrm{t}}$ approaches a diagonal matrix and therefore the two solutions converge.

Now, returning to the 16-element circular array with diameter of $D=\lambda / 2$, we compute the capacity averaged over the 500 channel realizations as a function of the threshold $Q$ factors $Q_{\mathrm{t} 0}=Q_{\mathrm{r} 0}$. Fig. 5 plots this capacity for both thermal noise and an isotropic external noise field using the water-filling and uninformed transmitter (Tx) solutions. The jumps in the capacity occur when the threshold is increased enough to increase the dimensionality of $\hat{\xi}_{\mathrm{t}}=\hat{\xi}_{\mathrm{r}}$. As expected, the water-filling solution that exploits channel state information at the transmitter is larger than the capacity for the uninformed transmitter, although the difference at this large SNR of $20 \mathrm{~dB}$ is relatively small [11]. Also, as $Q_{\mathrm{r} 0}$ increases, the receiver can use superdirectivity to improve the received SNR for the case of isotropic

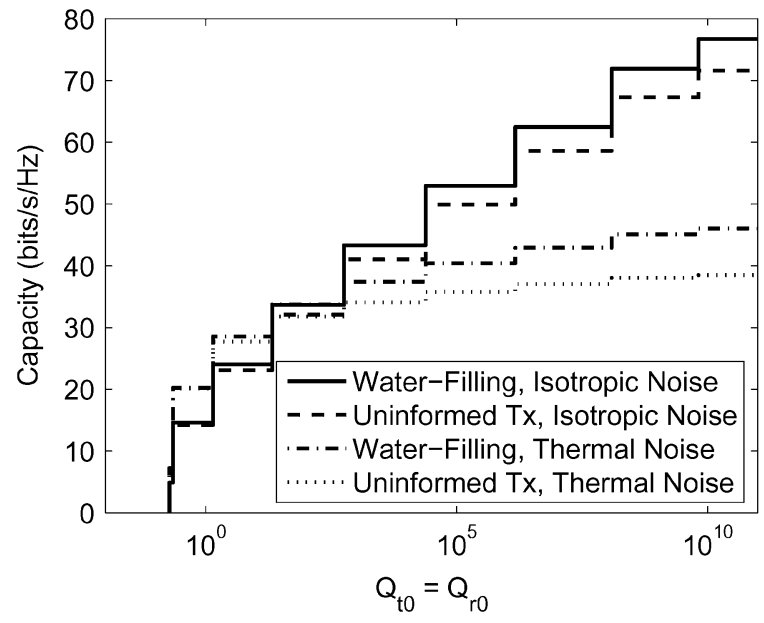

Fig. 5. Capacity (averaged over 500 channel realizations) for a 16-element circular array with diameter $D=\lambda / 2$ as a function of $Q_{\mathrm{t} 0}=Q_{\mathrm{r} 0}$ for different capacity solutions and noise models.

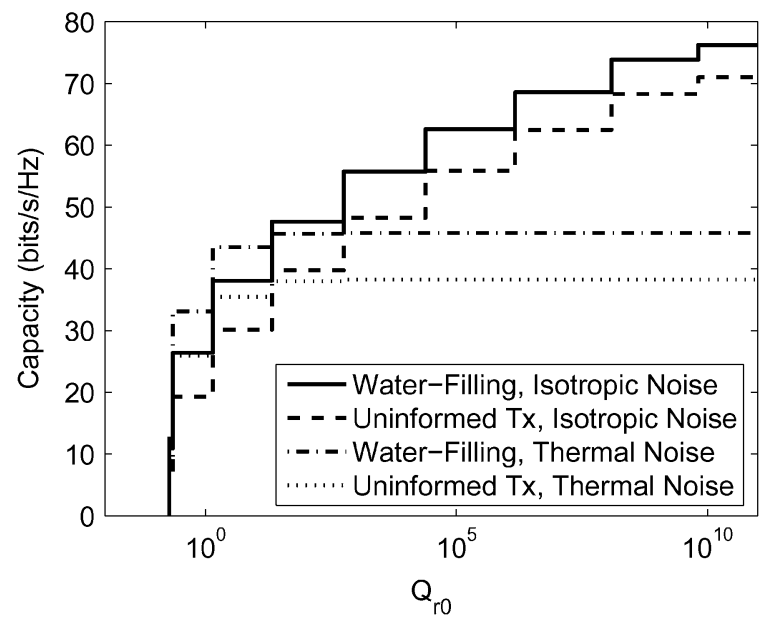

Fig. 6. Capacity (averaged over 500 channel realizations) for a 16-element circular array with diameter $D=\lambda / 2$ as a function of $Q_{\mathrm{r} 0}$ with $Q_{\mathrm{t} 0}=10^{10}$ for different capacity solutions and noise models.

noise. However, superdirectivity does not significantly enhance the capacity for thermal noise, leading to reduced capacity for this noise model.

Fig. 6 shows identical results for the case when $Q_{\mathrm{r} 0}$ is varied while $Q_{\mathrm{t} 0}=10^{10}$. These results reinforce the observation that the capacity for thermal noise is relatively insensitive to $Q_{\mathrm{r} 0}$ since superdirectivity does not play a large role in determining the SNR for this scenario. However, the effect of superdirectivity for the case of isotropic noise is dramatic, as expected. Figs. 5 and 6 both highlight the significant impact that superdirectivity can have on the channel capacity.

Fig. 7 plots the capacity as a function of $Q_{\mathrm{t} 0}=Q_{\mathrm{r} 0}$ for 4and 16-element linear arrays with length $L=\lambda / 2$. An isotropic noise model is assumed in all computations. This plot reveals that, if the antenna apertures remain fixed, including more elements within the apertures only increases the capacity if the system is allowed to use superdirectivity. Using studies such as this, designers can assess the practical upper bound for the number of elements to use on small devices. 


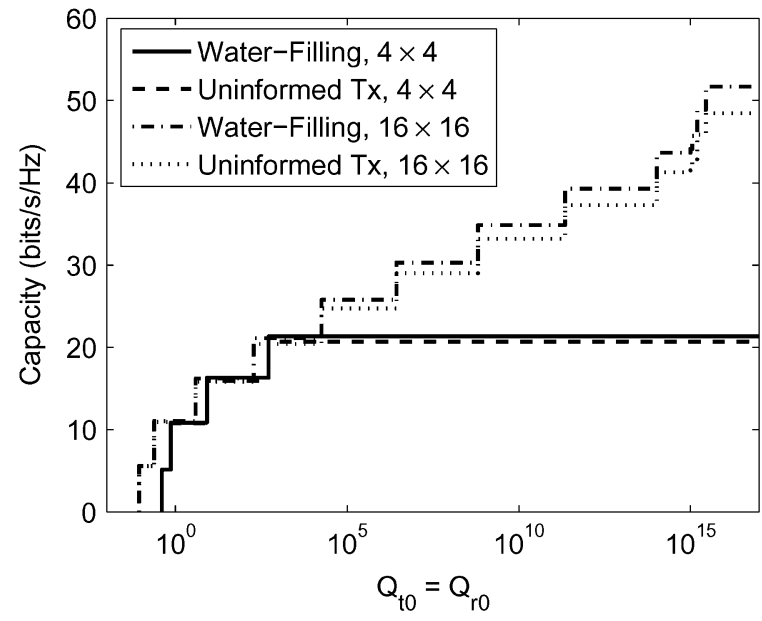

Fig. 7. Capacity (averaged over 500 channel realizations) for 4- and 16-element linear arrays with length $L=\lambda / 2$ as a function of $Q_{\mathrm{t} 0}=Q_{\mathrm{r} 0}$ for different capacity solutions and isotropic noise.
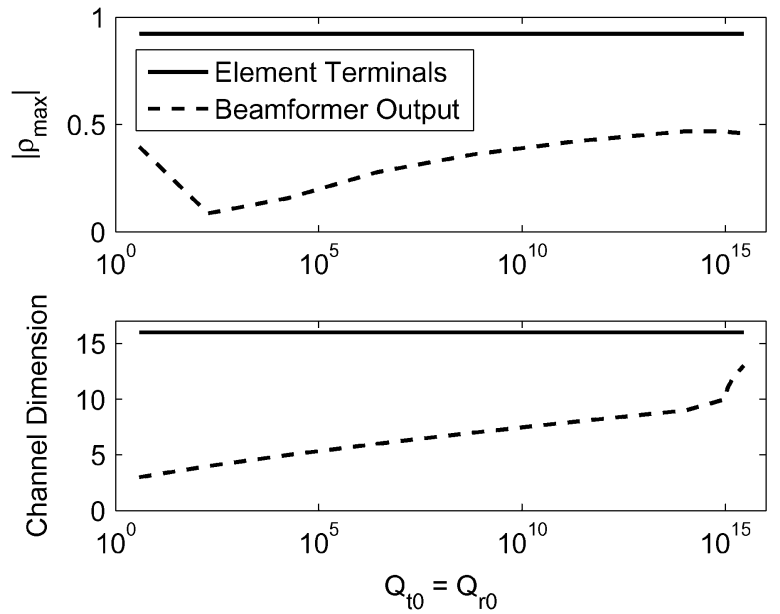

Fig. 8. Magnitude of the maximum correlation observed between array elements (or beams) and number of used elements (or beams) for a 16-element linear array with length $L=\lambda / 2$ as a function of $Q_{\mathrm{t} 0}=Q_{\mathrm{r} 0}$.

Finally, we compute the correlation coefficients for the 16-element linear array with length $L=\lambda / 2$ at the terminals of the antenna elements (channel matrix $\mathbf{H}$ ) and the terminals of the beamformers (channel matrix $\hat{\mathbf{H}}$ ). For the receiver, we then search over all shifts $p$ in (26) to find the value of $\rho_{\mathrm{r}, p}$ with the largest magnitude, which we will denote as $\rho_{\max }$. Fig. 8 plots the magnitude $\left|\rho_{\max }\right|$ as a function of the threshold superdirectivity $Q$ factor. The plot also shows the number of beams allowed by the beamformer under the superdirectivity constraint (which is simply the number of array elements when $\mathbf{H}$ is used). These results show that the beamformer always reduces the correlation relative to that observed at the antenna terminals. They also reveal that when the superdirectivity constraint significantly limits the number of beams, increasing the amount of allowable superdirectivity reduces the correlation due to reduced beam overlap (increased beam orthogonality). However, as the number of allowed beams increases, the likelihood that they will have some overlap also increases, which leads to the increasing correlation observed. Identical conclusions result from examining the transmit correlation.

\section{Alternate Signal Formulation}

The developments in Section II illustrate how superdirectivity can be enabled in MIMO communications. It is important to recognize, however, that there are other analysis approaches that also allow superdirectivity effects in the solution. For example, consider a transmitting array whose terminal impedance is described by an impedance matrix $\mathbf{Z}_{\mathrm{tt}}$, which, due to electromagnetic coupling, is in general full. For a transmit current vector $\mathbf{i}$, the radiated power averaged over one sinusoidal cycle becomes [15]

$$
\begin{aligned}
p_{\text {rad }} & =\frac{1}{2} \operatorname{Re}\left\{\mathbf{i}^{\dagger} \mathbf{Z}_{\mathrm{tt}} \mathbf{i}\right\} \\
& =\frac{1}{4}\left(\mathbf{i}^{\dagger} \mathbf{Z}_{\mathrm{tt}} \mathbf{i}+\mathbf{i}^{\dagger} \mathbf{Z}_{\mathrm{tt}}^{\dagger} \mathbf{i}\right)=\mathbf{i}^{\dagger} \underbrace{\left[\frac{\mathbf{Z}_{\mathrm{tt}}+\mathbf{Z}_{\mathrm{tt}}^{\dagger}}{4}\right]}_{\mathbf{Z}_{\mathrm{t}}} \mathbf{i} .
\end{aligned}
$$

Since for most arrays $\mathbf{Z}_{\mathrm{tt}}=\mathbf{Z}_{\mathrm{tt}}^{T}$, we may also write $\mathbf{Z}_{\mathrm{t}}=$ $\operatorname{Re}\left\{\mathbf{Z}_{\mathrm{tt}}\right\} / 2$. If the computation of the impedance matrix $\mathbf{Z}_{\mathrm{tt}}$ and the radiation patterns $\mathbf{e}_{\mathrm{t} n}\left(\Omega_{\mathrm{t}}\right)$ are performed properly, the matrix $\mathbf{Z}_{\mathrm{t}}$ constructed from (17) will be identical to that computed from (36).

While this formulation stems from a somewhat different model of the communication channel, the effective results are identical. The framework developed in Section II is therefore equally valid for this alternate signal formulation based on coupled antennas.

\section{CONCLUSION}

This paper has demonstrated that, when an appropriate constraint is placed on the radiated power of a MIMO system or when the receiver noise has certain characteristics, superdirectivity behavior impacts the capacity of the system. Because superdirectivity performance is typically impractical to achieve, modified capacity formulations for the cases of informed and uninformed transmitters have been developed that allow computation of the capacity under the constraint that the superdirectivity must remain below a predetermined threshold. Application of the framework to the capacity of MIMO systems with uniform circular and linear arrays has revealed that limiting the superdirectivity can have a dramatic impact on the achievable MIMO performance.

\section{REFERENCES}

[1] G. J. Foschini and M. J. Gans, "On limits of wireless communications in a fading environment when using multiple antennas," Wireless Personal Commun., vol. 6, pp. 311-335, Mar. 1998.

[2] G. G. Raleigh and J. M. Cioffi, "Spatio-temporal coding for wireless communication," IEEE Trans. Commun., vol. 46, no. 3, pp. 357-366, Mar. 1998.

[3] M. Uzsoky and L. Solymar, "Theory of super-directive linear antennas," Acta Phys., vol. 6, pp. 185-204, 1956.

[4] R. F. Harrington, "Antenna excitation for maximum gain," IEEE Trans. Antennas Propag., vol. AP-13, pp. 896-903, Nov. 1965.

[5] Y. T. Lo, S. W. Lee, and Q. H. Lee, "Optimization of directivity and signal-to-noise ratio of an arbitrary antenna array," Proc. IEEE, vol. 54, pp. 1033-1045, Aug. 1966.

[6] S. M. Sanzgiri and J. K. Butler, "Constrained optimization of the performance indices of arbitrary array antennas," IEEE Trans. Antennas Propag., vol. AP-19, pp. 493-498, Jul. 1971. 
[7] L. P. Winkler and M. Schwartz, "A fast numerical method for determining the optimum SNR of an array subject to a Q factor constraint," IEEE Trans. Antennas Propag., vol. AP-20, pp. 503-505, Jul. 1972.

[8] J. A. Kong, Electromagnetic Wave Theory. New York: Wiley, 1990.

[9] T. K. Moon and W. C. Stirling, Mathematical Methods and Algorithms for Signal Processing. Englewood Cliffs, NJ: Prentice-Hall, 2000.

[10] H. L. Van Trees, Optimum Array Processing: Part IV of Detection, Estimation, and Modulation Theory. New York: Wiley Interscience, 2002.

[11] M. A. Khalighi, J. Brossier, G. Jourdain, and K. Raoof, "Water filling capacity of Rayleigh MIMO channels," in Proc. 2001 IEEE 12th Int. Symp. Personal, Indoor Mobile Radio Comm., vol. 1, San Diego, CA, Sep. 30-Oct. 3 2001, pp. 155-158.

[12] T. M. Cover and J. A. Thomas, Elements of Information Theory. New York: Wiley, 1991.

[13] J. W. Wallace and M. A. Jensen, "Modeling the indoor MIMO wireless channel," IEEE Trans. Antennas Propag., vol. 50, no. 5, pp. 591-599, May 2002.

[14] J. W. Wallace, M. A. Jensen, A. L. Swindlehurst, and B. D. Jeffs, "Experimental characterization of the MIMO wireless channel: Data acquisition and analysis," IEEE Trans. Wireless Commun., vol. 2, pp. 335-343, Mar. 2003.

[15] J. W. Wallace and M. A. Jensen, "Mutual coupling in MIMO wireless systems: A rigorous network theory analysis," IEEE Trans. Wireless Commun., vol. 3, pp. 1317-1325, Jul. 2004.

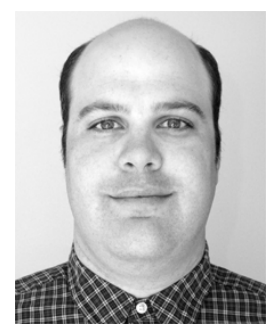

Matthew L. Morris received the B.S. degree in physics from Brigham Young University, Provo, UT, in 2000 , where he is currently working toward the Ph.D. degree in electrical engineering.

His research interests include multiple-input multiple-output communication with an emphasis on channel capacity.

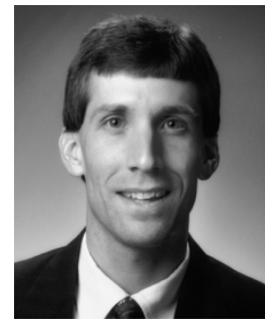

Michael A. Jensen (S'93-M'95-SM'01) received the B.S. (summa cum laude) and M.S. degrees in electrical engineering from Brigham Young University (BYU), Provo, UT, in 1990 and 1991, respectively, and the Ph.D. degree in electrical engineering from the University of California, Los Angeles (UCLA), in 1994.

From 1989 to 1991, he was a Graduate Research Assistant in the Lasers and Optics Laboratory, BYU. From 1991 to 1994 , he was a Graduate Student Researcher in the Antenna Laboratory, UCLA. Since 1994, he has been at the Electrical and Computer Engineering Department, BYU, where he is currently a Professor. His main research interests include antennas and propagation for personal communications, microwave circuit design, radar remote sensing, numerical electromagnetics, and optical fiber communications.

Dr. Jensen is a Member of Eta Kappa Nu and Tau Beta Pi. In 1990, he received a National Science Foundation Graduate Fellowship. He was awarded the best student paper award at the 1994 IEEE International Symposium on Antennas and Propagation, and received the Harold A. Wheeler Applications Prize Paper Award in the IEEE TRANSACTIONS ON ANTENNAS AND PROPAGATION in 2002. He currently serves on the Joint Meetings Committee for the IEEE Antennas and Propagation Society and as an Associate Editor for the IEEE TRANSACTIONS on ANTENNAS AND PROPAGation. He was the Technical Program Chair for the 2000 IEEE International Symposium on Antennas and Propagation and the General Co-Chair for the IEEE Topical Conference on Wireless Communications in 2003.

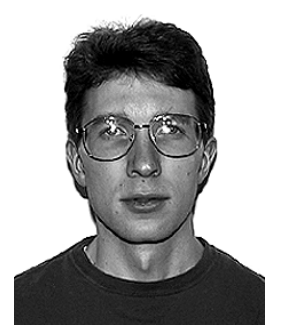

Jon W. Wallace, (S'99-M'03) received the B.S (summa cum laude) and Ph.D. degrees in electrical engineering from Brigham Young University (BYU), Provo, UT, in 1997 and 2002, respectively.

From 1995 to 1997, he worked as an Associate of Novell, Incorporated, Provo, UT. During 1997, he was a Member of Technical Staff for Lucent Technologies, Denver, CO. From 1998 to 2002, he worked as a Graduate Student Researcher in the BYU Wireless Communications Laboratory where he is currently a Research Associate. From 2002 to 2003, he visited the Technical University of Vienna Mobile Communications Group. His research interests include wireless channel sounding and modeling, optical device modeling, and remote sensing.

Dr. Wallace received the National Science Foundation Graduate Fellowship in 1999 and the Harold A. Wheeler Applications Prize Paper Award in the IEEE TRANSACTIONS ON ANTENNAS AND PROPAGATION in 2002. 